\title{
ON MONOMIALS AND HAYMAN'S PROBLEM
}

\author{
BY ZHAN-LIANG ZHANG AND WEI LI
}

\section{Introduction and main results}

Let $f(z)$ be a meromorphic function in the plane. We shall, for brevity, write / insted of $f(z)$. It is assumed that the reader is familiar with the notations of Nevanlinna theory (see, for example [1]). Throughout this paper we denote by $S(r, /)$, as usual, any function satisfying

$$
S(r, f)=o(T(r, /))
$$

as $r \rightarrow \infty$, possibly outside a set of $r$ value of finite linear measure and $N_{1}(r, f)$ and $N_{2}(r, /)$ count only the simple and multiple poles of / respectively.

L. R. Sons ([5]) has considered the monomial of form

$$
\psi^{=} f^{n_{0}}\left(f^{\prime}\right)^{n_{1}} \ldots\left(f^{(k)}\right)^{n_{k}}
$$

where $n_{0}, n_{1}, \cdots, n_{k}$ are non-negative integers. The following result is proved. with

THEOREM A. (i) If $f$ is a transcendental meromorphacfunction in the plane

$$
N_{11}\left(r, \frac{i}{f}\right)=S(r, /)
$$

and $\psi$ has the form (1) where $n_{0} \geqq 1, n_{k} \geqq 1, n_{\imath} \geqq 0$ for $\imath \neq \theta, k$ and if

$$
2^{k}\left(2 n_{0}+23(1+i) n_{\imath}\right)<\left(2^{k}+2 n_{0}-1\right)\left(233(1+i) n_{\imath}\right)
$$

then $\delta(c, \phi)<1$ for $c \neq 0$, $\infty$.

(ii) // / is a transcendental meromorphic function in the plane and $\phi$ has the form (1) where $n_{0} \geqq 2, n_{k} \geqq 1, n_{\imath} \geqq 0$ for $i \neq 0, k$, and if

$$
2^{k}\left(n_{0}+\sum_{\imath=0}^{k}(1+i) n_{\imath}\right)<\left(2^{k}+n_{0}-1\right)\left(\sum_{\imath=0}^{k}(1+i) n_{\imath}\right)
$$

then $\delta(c, \phi)<1$ for $c \neq 0, \infty$.

The assumption of Theorem A can be weakened. For $n_{0} \geqq 2$ N. Steinmetz ([7]) proved the following theorem :

Received January 9, 1991 Revised April 9, 1992. 
THEOREM B. Let $f$ be a transcendental meromorphicfunction in the plane and $\psi$ has the form (1). // $n_{0} \geqq 2, n_{1}+\cdots+n_{k} \geqq 1$, then

for $c \neq 0, \infty$.

$$
\limsup _{r \rightarrow \infty} \frac{\vec{N}\left(r, \frac{1}{\psi(-r}\right)}{\mathrm{T}(\mathrm{r}, \bar{\varphi})}>\mathbf{O}
$$

In this paper we use a modified version of Steinmetz's proof to consider the case of $n_{0}=1$ and prove condition (2) is not necessary. The result is the following :

THEOREM 1. Let $f$ be a transcendental meromorphic function in the plane with

$$
N_{1}\left(r, \frac{1}{f}\right)=S(r, f)
$$

and let

$$
\phi=f\left(f^{\prime}\right)^{n_{1}}\left(f^{\prime \prime}\right)^{n_{2}} \cdots\left(f^{(k)}\right)^{n_{k}}
$$

where $n_{1}, n_{2}, \cdots, n_{k}$ are non-negative integers. If $n_{1} \geqq 1$ then

for $c \neq 0, \infty$.

$$
\limsup _{r \rightarrow \infty} \bar{N}\left(r, \frac{1}{\psi-\overline{-}^{r}}\right)>0
$$

Obviously, Theorem 1 improves Sons's result.

Let / be a transcendental meromorphic function in the plane. W. K. Hayman ([2]) and E. Mues ([4]) proved respectively if $n \geqq 3$ and $n=2$ then $f^{n} f^{\prime}$ assumes all values except possibly zero infinitely often. The case $n=1$ is still open (W. K. Hayman [3], Problem 1.19), but our Theorem 1 enables us to obtain the following theorem:

THEOREM 2. Let $f$ be a transcendental meromorphic function in the plane with $N_{1}(r, 1 / f)=S(r, /)$. Then $f f$ assumes all values except possibly zero infinitely often.

\section{Preliminary results and lemmas}

For the proof of theorem we introduce some results on algebroid functions (cf. [8]).

The solution $w-w(z)$ of the functional equation

$$
a_{n}(z) w^{n}+\cdots+a_{0}(z)=0
$$

is called an algebroid function, where $a_{n}(z), \cdots, a_{0}(z)$ are meromorphic functions, $n$ is a positive integer. 
LEMMA 1 ([8]). // $a_{n}(z) \neq 0$, then equation (6) has at least one solution.

Obviously, meromorphic functions are algeoroid.

A polynomial in $w$ and their derivatives of the form

$$
Q[w]=\sum_{j=1}^{l} a_{j}(z) w^{l_{0}(j)}\left(w^{\prime}\right)^{l_{1}^{(j)}} \cdots\left(w^{(k j)}\right)^{l_{k j}^{(j)}}
$$

is called a differential polynomial in $w$, where $a_{j}(z)(j=1, \cdots, /)$ are meromorphic functions satisfying

$$
T(r, \mathrm{fl},)=\mathrm{S}(\mathrm{r}, w), \quad \jmath=1, \cdots, l .
$$

If $Q[w]$ has only one term, it is called a (differential) monomial in $w$. We denote $(d / d z) Q[w]$ as $Q^{\prime}[w]$.

If (8) is replaced with $m\left(r, a_{j}\right)=S(r, w)$, then $Q[w]$ is called a quasi-differential polynomial in $w$. The following lemma on quasi-differential polynomials is essentially due to $\mathrm{He}$ Yu-Zhan and Xiao Xiu-Zhi $([8,9])$

LEMMA 2. Let $w$ be a nonconstant algebroid function, $Q_{1}[w]$ and $Q_{2}[w]$ be quasi-differentrabolynomials in $w$ and $n$ be a positive integer. If

$$
w^{n} Q_{1}[w]=Q_{2}[w]
$$

and $n \geqq \gamma_{Q_{2}}$ then $m\left(r, Q_{1}[w]\right)=S(r, w)$, where $\gamma_{Q_{2}}$ is the degree of $Q_{2}[w]$.

LEMMA 3. Let $w$ be an algebroid function, $Q[w]$ be a differential polynomial in $w$, and $n$ be a positive integer. If

$$
w^{n} Q[w]=d \text { and } d \neq 0 \text { is Const, }
$$

then $w \equiv$ Const.

Proof. Obviously, $Q[w] \not \equiv 0$. Suppose $u \neq$ Const, then Lemma 2 yields $m(r, Q[w])=S(r, w)$.

The poles of $w$ are not any poles of $Q[w]$ by (9). Combining (7) and (8), we get

Thus

$$
N(r, Q[w])=S(r, w)
$$

and

$$
T(r, Q[w])=S(r, w)
$$

$$
\begin{aligned}
n T(r, w) & =T\left(r, \frac{1}{Q[w]}\right)+O(1) \\
& =T(r, Q[w])+O(1)=S(r, w) .
\end{aligned}
$$

This is impossible. Thus Lemma 3 is proved. 


\section{Proof of Theorem 1}

Suppose that there is some $c \neq 0, \infty$, such that

$$
\limsup _{r \rightarrow \infty} \frac{\bar{N}\left(r, \frac{1}{\phi-c}\right)}{T(r, \phi)}=0 .
$$

Since $T(r, \phi)=O(T(r, /))$, we get

$$
\bar{N}\left(r, \frac{1}{\psi-c}\right)=S(r, f) .
$$

Without loss of the generality, we may assume that $c=1$. Set

and

$$
Q\left[f^{\prime}\right]=\left(f^{\prime}\right)^{n_{1}} \cdots\left(f^{(k)}\right)^{n_{k}}
$$

Then

$$
F=\phi-1=f Q\left[f^{\prime}\right]-1 .
$$

$$
\bar{N}\left(r, \frac{i}{F}\right)=S(r, f) .
$$

Obviously, $F \not \equiv 0$. By (10) we obtain

$$
f Q^{\prime}\left[f^{\prime}\right]+f^{\prime} Q\left[f^{\prime}\right]=f Q\left[f^{\prime}\right] \frac{F^{\prime}}{F}-\frac{F^{\prime}}{F} .
$$

That is,

$$
f a(z)=-\frac{F^{\prime}}{F}
$$

where

$$
a(z)=Q^{\prime}\left[f^{\prime}\right]+\frac{f^{\prime}}{f} Q\left[f^{\prime}\right]-Q\left[f^{\prime}\right] \frac{F^{\prime}}{F}
$$

is a quasi-differential polynomial in $/$, since $m(r, f / f)=S(r, /)$ and $m\left(r,-F^{\prime} / F\right)$ $=S(r, /)$.

If $a(z) \equiv 0$, then $F \equiv$ Const. Further $f \equiv$ Const by Lemma 3 and (10). Hence $a(z) \not \equiv 0$.

From (12) and Lemma 2 we obtain

$$
m(r, a)=S(r, f) .
$$

Now we note that $a(z)$ can have poles only at the poles or zeros of / or the zeros of $\mathrm{F}$ by (13). Since $n_{1} \geqq 1$ and

$$
\frac{f^{\prime}}{f}-Q\left[f^{\prime}\right]=\frac{\left(f^{\prime}\right)^{n_{1}+1}}{f}\left(f^{\prime \prime}\right)^{n_{2}} \cdots\left(f^{(k)}\right)^{n_{k}}
$$

it is easily seen from (13) that the multiple zeros of / are not any poles of $a(z)$. On the other hand, by (12) the poles of / are not any poles of $a(z)$. 
Thus

$$
N(r, a) \leqq N_{1)}\left(r, \frac{1}{f}\right)+\bar{N}\left(r, \frac{1}{F}\right) .
$$

Together with above inequalities (4), (11) and (14), we get

$$
\mathrm{T}(\mathrm{r}, a)=S(r, /) \text {. }
$$

Dividing equation (12) by $a(z)$, we deduce

so that

$$
\begin{aligned}
m(r, f) & \leqq m\left(r, \frac{1}{a}\right)+m\left(r, \frac{F^{\prime}}{F}\right)+O(1) \\
& \leqq T(r, a)+S(r, f)
\end{aligned}
$$

$$
m(r, f)=S(r, f) \text {. }
$$

It is easily seen from (12) that $a(z)$ has a zero of multiplicity at least $q-1$ at any pole of / with order $q(\geqq 2)$. Thus, we have

$$
N_{c_{2}}(r, f) \leqq 2 N\left(r, \frac{1}{a}\right) \leqq 2 T(r, a)+O(1),
$$

so that

$$
N_{\iota_{2}}(r, f)=S(r, /)
$$

Thus / must have infinite simple poles.

Now we multiply (12) by $f Q\left[f^{\prime}\right]$ and (13) by / respectively and subtract. This gives

$$
a(z) Q\left[f^{\prime}\right] f^{2}+Q^{\prime}\left[f^{\prime}\right] f+Q\left[f^{\prime}\right] f^{\prime}-a(z) f=0 .
$$

Let $z_{0}$ be a simple pole of $/$, then $a\left(z_{0}\right) \neq 0, \infty$ by (12). We may write $f(z)$ and $a(z)$ near $z_{0}$ in the form

$$
f(z)=\frac{\bar{d}_{1}}{z \quad z_{0}}+\bar{d}_{0}+O\left(z-z_{0}\right)
$$

and

$$
a(z)=a\left(z_{0}\right)+a^{\prime}\left(z_{0}\right)\left(z-z_{0}\right)+O\left(\left(z-z_{0}\right)^{2}\right),
$$

where $\bar{d}_{1} \neq 0$ and $\bar{d}_{0}$ depend on $z_{0}$. Combining these with (18) we see that the coefficients $\bar{d}_{1}$ and $\bar{d}_{0}$ have the form

$$
\bar{c}_{1}^{\bar{T}}=z_{a\left(z_{0}\right)}, \quad \begin{array}{ccc}
\bar{u}_{0} & (\Gamma+1)^{2} & a^{\prime}\left(z_{0}\right) \\
\Gamma+2 & a^{2}\left(z_{0}\right)
\end{array}
$$

where $\Gamma=2 n_{1}+-+(k+1) n_{k}$. Thus if let

$$
d_{1}(z)=\frac{\Gamma+1}{a(z)}, \quad d_{0}=-\frac{(\Gamma+1)^{2}}{\Gamma+2} \frac{a^{\prime}(z)}{a^{2}(z)}
$$

then $d_{1}\left(z_{0}\right)=\bar{d}_{1}, d_{0}\left(z_{0}\right)=\bar{d}_{0}$ and 


$$
f(z)=\frac{d_{1}\left(z_{0}\right)}{z-z_{0}}+d_{0}\left(z_{0}\right)+O\left(z-z_{0}\right)
$$

for any simple pole $z_{0}$ of $/$. Furthermore $d_{1}(z)$ and $d_{0}(z)$ are meromorphic and

$$
T\left(r, \mathrm{rfO}+T\left(r, d_{0}\right)=S(r, f)\right.
$$

by (15). Here by using (16), (17) and Steinmetz's Lemma 2 (see [6, P 156]), we know that there exist the meromorphic functions $b_{0}(z), b_{1}(z), b_{2}(z)(\not \equiv)$ satisfying

such that

$$
\mathrm{T}\left(\mathrm{r}, b_{i}\right)=S(r, f), \quad(i=0,1,2)
$$

$$
f^{\prime}=b_{0}(z)+b_{1}(z) f+b_{2}(z) f^{2} .
$$

That is, / satisfies Riccati equation

$$
w^{\prime}=b_{0}(z)+b_{1}(z) w+b_{2}(z) w^{2} .
$$

Using (20) over and over again we deduce that

$$
f^{(j)}=j ! b_{2}^{3}(z) f^{3+1}+\quad, \quad j=1,2, \cdots
$$

are polynomials in /. Thus we may write

and

$$
Q\left[f^{\prime}\right]=P(z, f)
$$

$$
\begin{aligned}
F & =f Q\left[f^{\prime}\right]-1 \\
& =f P(z, f)-1,
\end{aligned}
$$

where $P(z, /)$ is a polynomial in / and the coefficients $\left\{\alpha_{i}\right\}$ are meromorphic functions satisfying

$$
T\left(r, \alpha_{i}\right)=S(r, f), \quad \imath=0,1,2, \cdots
$$

by (19).

Now we consider the function of $z, w$

$$
G(z, w)=w P(z, w)-1 .
$$

This is a polynomial in $w$ and satisfies the identity

$$
G(z, f(z)) \equiv F
$$

by (22). We will prove that the solution $w=w(z)$ of the functional equation

$$
G(z, w)=0
$$

satisfies Riccati equation (21) and so that

$$
w(z) Q\left[w^{\prime}(z)\right]-F(z, w(z)) \equiv 0 .
$$


We rewrite (12) in the form

$$
a(z) f F+F^{\prime} \equiv 0
$$

it follows that $H(z, f) \equiv 0$, where

$$
H(z, w)=a(z) w G(z, w)+G_{z}^{\prime}(z, w)+G_{w}^{\prime}(z, w)\left(b_{0}(z)+b_{1}(z) w+b_{2}(z) w^{2}\right)
$$

is a polynomial in $w$ and the coefficients $\left\{\beta_{i}\right\}$ are meromorphic functions satisfying

$$
\mathrm{T}\left(\mathrm{r}, \beta_{i}\right)=S(r, /)
$$

by (15), (19), (23) and (24). Hence $H(z, f) \equiv 0$ implies $H(z, w)=0$ for arbitrary complex $z$ and $w$. That is,

$$
a(z) w G(z, w)+G_{z}^{\prime}(z, w)+G_{w}^{\prime}(z, w)\left(b_{0}(z)+b_{1}(z) w+b_{2}(z) w^{2}\right)=0
$$

for arbitrary complex $z$ and $w$.

Let $w-w(z)$ be a solution of (25). Then there is a unique positive integer $\lambda$ such that

$$
G(z, w)=(w-w(z))^{2} G^{*}(z, w), \quad G^{*}(z, w(z)) \neq 0 .
$$

The equations (27) and (28) yield

$$
\begin{gathered}
(w-w(z))^{\lambda}\left(a(z) w G^{*}(z, w)+G_{z}^{* \prime}(z, w)+G_{w}^{* \prime}(z, w)\left(b_{0}(z)+b_{1}(z) w+b_{2}(z) w^{2}\right)\right. \\
-\lambda(w-w(z))^{\lambda-1}\left(w^{\prime}(z)-\left(b_{0}(z)+b_{1}(z) w+b_{2}(z) w^{2}\right)\right) G^{*}(z, w) \equiv 0 .
\end{gathered}
$$

Dividing by $(w-w(z))^{\lambda-1}$ and letting $w-w(z)$ we get the desired result that

$$
w^{\prime}(z) \equiv b_{0}(z)+b_{1}(z) w(z)+b_{2}(z) w^{2}(z) .
$$

By (26) the functional equation (25) has not any constant solution. On the other hand, by using Lemma 3 to (26) we know that the functional equation (25) has only constant solution. These imply that the functional equation (25) has not any solution. It contradicts Lemma 1 . Theorem 1 is proved.

\section{REFERENCES}

[ 1 ] W. K. HAYMAN, Meromorphic functions, Oxford, 1964.

[2] W. K. HAYMAN, Picard values of meromorphic functions and their derivatives, Ann. of Math., 70 (1959), 9-42.

[ 3 ] W. K. HAYMAN, Research problems in function theory, London University Press, 1967.

[4] E. MUES, Über ein Problem von Hayman, Math. Z., 164 (1979), 239-259.

[5] L.R. SONS, Deficiencies of monomials, Math. Z., 111 (1969), 53-68.

[6] N. Steinmetz, Ein Malmquistsher Satz fur algebraische Differentialgeichungen Zweiter Ordnung, Results in Math., Vol. 10 (1986).

[7] N. S'teinmetz, Über die Nullstellen von Differentialpolynomen, Math. Z., 176 
(1981), 255-264.

[ 8 ] YU-ZHAN HE AND Xiu-ZHI XIAO, Algebroid functions and ordinary differential equations, Beijing, 1988 .

[ 9] YU-ZHAN HE AND Xiu-ZHI XIAO, Single-valued meromorphic solutions and finite branched solutions of higher-order algebroid differential equations, Scientia Sinica, 6 (1983), 514-522.

DEPARTMENT OF MATHEMATICS

HEZE TEACHERS' COLLEGE

HEZE, SHANDONG 274015

P.R. CHINA 